\title{
Comparison between melanoma gene expression score and fluorescence in situ hybridization for the classification of melanocytic lesions
}

Eugen C Minca ${ }^{1,2}$, Rami N Al-Rohil ${ }^{3}$, Min Wang ${ }^{4}$, Paul W Harms ${ }^{4}$, Jennifer S Ko ${ }^{1,2}$, Angela M Collie ${ }^{1,2}$, Ivanka Kovalyshyn ${ }^{1,2}$, Victor G Prieto ${ }^{3,5}$, Michael T Tetzlaff ${ }^{3,6}$, Steven D Billings ${ }^{1,2}$ and Aleodor A Andea ${ }^{4}$

${ }^{1}$ Department of Pathology, Cleveland Clinic, Cleveland, OH, USA; ${ }^{2}$ Department of Dermatology, Cleveland Clinic, Cleveland, OH, USA; ${ }^{3}$ Department of Pathology, MD Anderson Cancer Center, The University of Texas, Houston, TX, USA; ${ }^{4}$ Department of Pathology, University of Michigan Medical Center, Ann Arbor, MI, USA; ${ }^{5}$ Department of Dermatology, MD Anderson Cancer Center, The University of Texas, Houston, TX, USA and ${ }^{6}$ Department of Tanslational and Molecular Pathology, MD Anderson Cancer Center, The University of Texas, Houston, TX, USA

\begin{abstract}
Melanoma accounts for most skin cancer-related deaths and has an increasing incidence. Accurate diagnosis and distinction from atypical nevi can be at times difficult using light microscopy alone. Fluorescence in situ hybridization (FISH) and melanoma gene expression score (myPath, Myriad Genetics) have emerged as ancillary tools to further aid in this differential diagnosis. Our aim in this study was to correlate FISH results, gene expression score, consensus histopathologic impression and clinical outcome on a series of 117 challenging melanocytic lesions collected from three separate institutions. The lesions were separated into two groups: 39 histopathologically unequivocal lesions (15 malignant, 24 benign) and 78 challenging lesions interpreted by expert consensus (27 favor malignant, 30 favor benign, and 21 ambiguous). Melanoma-FISH was performed using probes for 6p25, 11q13, 8q24, and 9p21/CEP9 and scored according to established criteria. Analysis by myPath gene expression score was performed and interpreted by the manufacturer as 'benign', 'indeterminate,' or 'malignant'. In the unequivocal group, melanoma-FISH and myPath score showed 97 and $83 \%$ agreement with the histopathologic diagnosis, respectively, with 93 and $62 \%$ sensitivity, 100 and $95 \%$ specificity, and $80 \%$ intertest agreement. In the challenging group, FISH and the myPath score showed 70 and $64 \%$ agreement with the histopathologic interpretation, respectively, with $70 \%$ inter-test agreement and similar sensitivities and specificities. The inter-test agreement was $73 \%$ overall, excluding indeterminate results. Discordant test results occurred in 27/117 cases from both unequivocal and challenging groups. Melanoma-FISH and gene expression score are valuable ancillary tools, though both have limitations and return discordant results in a subset of cases. Follow-up studies with more extensive clinical outcome data are warranted to establish the accuracy of these tests for the classification of melanocytic lesions.

Modern Pathology (2016) 29, 832-843; doi:10.1038/modpathol.2016.84; published online 13 May 2016
\end{abstract}

Melanoma is the most aggressive malignancy among the common forms of skin cancer and its incidence is on the rise. ${ }^{1}$ The significance of a positive diagnosis

Correspondence: Dr EC Minca, MD, PhD, Department Pathology, Cleveland Clinic, 9500 Carnegie Ave, L25, Cleveland, OH 44195, USA.

E-mail: mincae@ccf.org

Received 17 November 2015; revised 22 March 2016; accepted 24 March 2016; published online 13 May 2016 thus carries tremendous clinical implications. A benign (albeit atypical) lesion is considered to be cured with conservative excision, while malignant melanoma requires a significantly greater surgical intervention with associated morbidity and longterm surveillance. Not inconsequential is also the psychological burden for the patient carrying a malignant diagnosis with well-known lethal potential for the remainder of his or her life. Accurate diagnosis of melanocytic proliferations remains one 
of the most problematic areas in dermatopathology, as there is a subset of melanocytic lesions with an ambiguous histopathologic appearance, demonstrating some but not all of the features associated with common melanomas, making their accurate classification difficult. Indeed, a number of studies have shown significant diagnostic disagreement between expert dermatopathologists in their histologic interpretation of melanocytic lesions. ${ }^{2-5}$ Thus there is a critical need to develop and validate novel ancillary testing methodologies to improve diagnostic accuracy.

Fluorescence in situ hybridization (FISH) probing genomic loci known to harbor numerical aberrations in melanoma and evaluation of expression levels of genes differentially expressed in melanoma vs nevus have emerged as adjunct molecular tools to aid in the interpretation of diagnostically challenging melanocytic lesions. Melanoma-FISH is a well-established commercially available test. ${ }^{6-8}$ The original format used three probes for chromosome 6 and one for chromosome 11. A genotype is consistent with melanoma if any one of four previously established criteria is met in a threshold percentage of tumor cells: absolute or relative gain of $6 \mathrm{p} 25$, relative loss of $6 q 23$ or absolute gain of $11 \mathrm{q} 13 .{ }^{6}$ The first study to interrogate the utility of FISH demonstrated a sensitivity of $\sim 87 \%$ and a specificity of $\sim 95 \%$ in the distinction of morphologically unequivocal melanoma from nevi. ${ }^{6}$ Numerous subsequent confirmatory 'proof of principle' studies validated the diagnostic utility of FISH in many different diagnostic settings, reporting a $75-100 \%$ sensitivity and $89-100 \%$ specificity of the assay in differentiating histologically unequivocal melanoma and nevi. ${ }^{6,9-15}$ However, most of the earliest proof of principle studies utilized histopathologically unambiguous nevi and melanomas to demonstrate the utility of FISH as an ancillary diagnostic test, yet FISH is predominantly deployed in the setting of morphologically ambiguous melanocytic tumors, where ancillary testing most usefully informs the diagnosis. The diagnostic utility of FISH is controversial in the setting of such ambiguous melanocytic lesions, where the overall reported sensitivity lies between 43 and $100 \%$ and the specificity between 33 and 83\% using clinical behavior or expert histopathologic review as the gold standard.$^{6,16-19}$ Of note, within spitzoid tumors, FISH testing has a low sensitivity in detecting spitzoid melanoma around $70 \%$. Thus, to improve on the diagnostic sensitivity and specificity and to overcome the limitations imposed by tetraploidy, probes for 9p21, a region homozygously deleted in a subset of spitzoid melanomas, and for $8 \mathrm{q} 24$, the gain of which is associated with poorer outcome, were integrated into the standard FISH panel to increase the diagnostic sensitivity and specificity of the assay and add prognostic relevance. ${ }^{20}$

The gene expression-based test myPath (Myriad Genetics) measures the mRNA expression of 23 genes by quantitative RT-PCR. ${ }^{21,22}$ The functionality of the gene products measured is diverse and includes one gene specific to melanocytic differentiation, eight genes implicated in immune signaling pathways, five genes with multifunctional roles, and nine housekeeping genes. A proprietary algorithm measures the relative expression levels of these genes in a given melanocytic lesion and calculates a gene expression score that can differentiate benign and malignant lesions. A negative score $(-16.7$ to -2.1) supports a benign diagnosis, while a positive score (0.0-11.1) supports a diagnosis of melanoma. Because there is an overlap in scores between the spectrum of malignant and benign lesions in the validation set, an indeterminate category was created with scores between -2 and -0.1 , representing $\sim 10 \%$ of cases. Excluding the indeterminate cases, the assay has a reported sensitivity of $90 \%$ and a specificity of $91 \%$ in distinguishing unambiguous benign from malignant melanocytic lesions. ${ }^{22}$ The presence of an indeterminate category is an important limitation, with no currently available data on the prognosis and clinical behavior of such cases. Furthermore, because this test has only recently become available, there is limited clinical experience with the operating characteristics of this assay, and comparative studies with other ancillary tests are lacking.

Here, we correlated clinical melanoma-FISH, myPath gene expression score, and consensus histopathologic impression on a series of 117 melanocytic lesions collected from three tertiary referral centers.

\section{Materials and methods}

\section{Specimens}

The study included 117 formalin-fixed paraffin embedded skin biopsies: 67 from University of Michigan Medical Center (Ann Arbor, MI, USA), 35 from Cleveland Clinic (Cleveland, OH, USA), and 15 from MD Anderson Cancer Center (Houston, TX, USA) (Supplementary Data). The total of 117 cases was composed of two distinct groups.

The first group of 39 samples was considered histopathologically unequivocal with a final diagnosis rendered as either benign (nevus, $n=24$ ) or malignant (melanoma, $n=15$ ) by a single pathologist (AAA or SDB). The benign lesions included banal nevi $(n=3)$, dysplastic nevi $(n=10)$, Spitz nevi $(n=8$, of which 4 desmoplastic), cellular blue nevi $(n=2)$, and Reed nevus $(n=1)$. The malignant lesions included superficial spreading melanomas $(n=12)$, nodular melanoma $(n=1)$, and nevoid melanoma $(n=1)$.

The second group comprised the remaining 78 cases which on initial evaluation at each institution were deemed to exhibit challenging histopathologic characteristics, warranting further molecular investigation. The cases in this group were then 
Table 1 Case distribution based on histopathologic interpretation with associated molecular test results

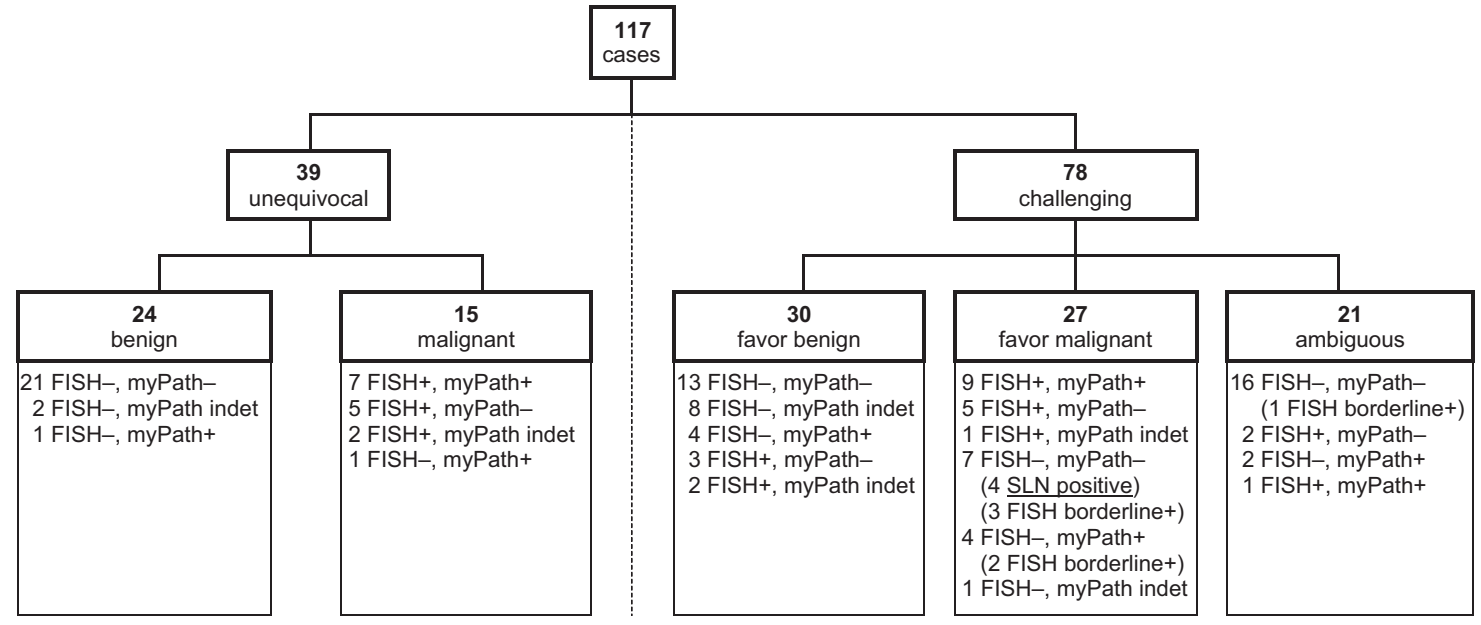

FISH—Fluorescence in situ hybridization; Indet—indeterminate; SLN—sentinel lymph node.

re-reviewed by all three expert pathologists (AAA, SDB, and MTT) and a consensus interpretation was rendered as 'favor benign' $(n=30)$, 'ambiguous' $(n=21)$, or 'favor malignant' $(n=27)$ (Table 1$)$. This group included spitzoid lesions $(n=37)$, lesions with the differential dysplastic nevus/nevoid melanoma $(n=35)$, lesions with the differential traumatized nevus/regressed melanoma $(n=3)$, and blue nevus-like lesions $(n=3)$.

\section{FISH}

Melanoma-FISH was performed with commercially available platforms as follows. On the cases from University of Michigan Medical Center and Cleveland Clinic, testing was performed using Vysis Melanoma-FISH Probes (Abbott Molecular, Des Plaines, IL, USA) for 6p25 (RREB1), CEP6, 6q23 (MYB), 11q13 (CCND1), 8q24 (MYC), 9p21 (CDKN2A), and CEP9 (centromeric reference), according to the manufacturer's instructions. On the cases from MD Anderson Cancer Center, testing was performed at NeoGenomics Laboratories using NeoSITE Melanoma probes (NeoGenomics Laboratories, Fort Myers, FL, USA) using a restricted protocol that did not include 6q23.

All cases were interpreted as positive for a genotype consistent with melanoma, if any of the following abnormalities was present in more than $29 \%$ of the cells: gain of $6 \mathrm{p} 25$, gain of $11 \mathrm{q} 13$, gain of $8 q 24$, or homozygous loss of 9p21, according to previously published criteria. ${ }^{20}$

For the analysis in the last section of the Results only (Table 5), a 'borderline positive' subcategory was created based on less stringent cutoffs used by NeoGenomics Laboratories as follows: gain of $6 \mathrm{p} 25$ in $17-29 \%$ of cells, gain of 11 q13 in $20-29 \%$ of cells, gain of 8 q24 in 11-29\% of cells, or homozygous loss of 9 p21 in $11-29 \%$ of cells ${ }^{23}$ (http://www.neoge nomics.com/neosite-melanoma.htm).

\section{Gene Expression Score}

All samples underwent analysis with the myPath melanoma gene expression score (Myriad Genetics, Salt Lake City, UT, USA) and were classified as 'benign', 'indeterminate,' or 'malignant' based on the cutoffs established by the manufacturer. ${ }^{21,22}$

\section{Statistical Analysis}

Fisher exact test calculations for comparing the concordance between melanoma-FISH and gene expression score were performed with GraphPad Prism (GraphPad Software, La Jolla, CA, USA).

\section{Results}

\section{Test Performance in the Unequivocal Case Group}

We first evaluated the performance of melanomaFISH and gene expression score in the group of 39 histopathologically unequivocal cases. MelanomaFISH had 97\% overall agreement with the morphologic diagnosis (93\% sensitivity, 100\% specificity) (Table 2a). Gene expression score showed $83 \%$ agreement with the histopathologic diagnosis (62\% sensitivity, 97\% specificity) (Table 2a). Four cases $(10 \%)$ were classified as 'indeterminate' by myPath score. Finally, when comparing gene expression score with melanoma-FISH, the two tests showed $80 \%$ overall agreement $(P=0.006)$ (Table 3a).

\section{Test Performance in the Challenging Case Group}

We next compared the performance of melanomaFISH and gene expression score in the second group of 78 histopathologically challenging lesions. Melanoma-FISH showed a $70 \%$ overall agreement 
Table 2 Correlation of melanoma-FISH and myPath gene expression score with the expert histopathologic interpretation

\begin{tabular}{|c|c|c|c|c|c|c|c|}
\hline & \multicolumn{2}{|c|}{ Histopathology } & \multirow{2}{*}{ Total } & & & & \multirow{2}{*}{$95 \% C I$} \\
\hline & Malignant & Benign & & & & & \\
\hline \multicolumn{8}{|c|}{ (A) Unequivocal cases } \\
\hline \multicolumn{8}{|c|}{ FISH } \\
\hline Positive & 14 & 0 & 39 & \multicolumn{2}{|c|}{ Sensitivity } & $93 \%$ & $0.66-0.99$ \\
\hline Negative & 1 & 24 & & \multirow{2}{*}{\multicolumn{2}{|c|}{$\begin{array}{l}\text { Specificity } \\
\text { Agreement }\end{array}$}} & \multirow{2}{*}{$100 \%$} & $0.83-1.00$ \\
\hline & & & & & & & \\
\hline \multicolumn{8}{|l|}{ myPath } \\
\hline Malignant & 8 & 1 & 39 & \multicolumn{2}{|c|}{ Sensitivity } & $62 \%$ & $0.32-0.85$ \\
\hline Benign & 5 & 21 & & \multicolumn{2}{|c|}{ Specificity } & $95 \%$ & $0.75-0.99$ \\
\hline \multirow[t]{2}{*}{ Indeterminate } & 2 & 2 & & \multicolumn{2}{|c|}{ Agreement } & $83 \%$ & \\
\hline & Favor malignant & Favor benign & Ambiguous & Total & & & $95 \% C I$ \\
\hline \multicolumn{8}{|c|}{ (B) Challenging cases } \\
\hline \multicolumn{8}{|l|}{ FISH } \\
\hline Positive & 15 & 5 & 3 & \multirow[t]{3}{*}{78} & Sensitivity & $56 \%$ & $0.36-0.74$ \\
\hline Negative & 12 & 25 & 18 & & Specificity & $83 \%$ & $0.65-0.94$ \\
\hline & & & & & Agreement & $70 \%$ & \\
\hline \multicolumn{8}{|l|}{ myPath } \\
\hline Malignant & 13 & 4 & 3 & \multirow{3}{*}{78} & Sensitivity & $52 \%$ & $0.32-0.72$ \\
\hline Benign & 12 & 16 & 18 & & Specificity & $80 \%$ & $0.56-0.93$ \\
\hline Indeterminate & 3 & 9 & 0 & & Agreement & $64 \%$ & \\
\hline \multicolumn{8}{|l|}{ (C) All cases } \\
\hline \multicolumn{8}{|l|}{ FISH } \\
\hline Positive & 29 & 5 & 3 & \multirow[t]{3}{*}{117} & Sensitivity & $69 \%$ & $0.53-0.82$ \\
\hline Negative & 13 & 49 & 18 & & Specificity & $91 \%$ & $0.79-0.97$ \\
\hline & & & & & Agreement & $81 \%$ & \\
\hline \multicolumn{8}{|l|}{ myPath } \\
\hline Malignant & 21 & 5 & 3 & \multirow[t]{3}{*}{117} & Sensitivity & $55 \%$ & $0.38-0.71$ \\
\hline Benign & 17 & 37 & 18 & & Specificity & $88 \%$ & $0.74-0.96$ \\
\hline Indeterminate & 5 & 11 & 0 & & Agreement & $73 \%$ & \\
\hline
\end{tabular}

A-unequivocal cases; B—challenging cases; C-all cases. Statistic parameters calculated on non-indeterminate cases.

Table 3 Correlation between myPath gene expression score and melanoma-FISH

\begin{tabular}{lrrrr}
\hline & \multicolumn{2}{c}{ FISH } & & \\
\cline { 2 - 2 } & Positive & Negative & & \\
& & & & \\
(A) Unequivocal cases & & & & \\
myPath & & & & \\
$\quad$ Malignant & 5 & 21 & & \\
$\quad$ Benign & 5 & 2 & & \\
Indeterminate & 2 & & & \\
(B) Challenging cases & & & & \\
myPath & & & & \\
$\quad$ Malignant & 10 & 10 & & \\
$\quad$ Benign & 10 & 36 & & \\
Indeterminate & 3 & 9 & & \\
& & & & \\
(C) All cases & & & & \\
myPath & 17 & 12 & 117 & \\
$\quad$ Malignant & 15 & 57 & & \\
$\quad$ Benign & 5 & 11 & & \\
$\quad$ Indeterminate & 5 & & & \\
\hline
\end{tabular}

A-unequivocal cases; B—challenging cases; C-all cases. Statistic parameters calculated on non-indeterminate cases. with the final interpretation (56\% sensitivity, $83 \%$ specificity), excluding 21 cases that remained interpreted as 'ambiguous' after expert review (Table $2 b$ ). Gene expression score showed $64 \%$ agreement with the consensus interpretation ( $52 \%$ sensitivity, $80 \%$ specificity), again after excluding the 21 'ambiguous' cases (Table $2 \mathrm{~b}$ ), as well as 12 cases $(15 \%)$ were classified as 'indeterminate' by myPath score. Lastly, when comparing gene expression score with melanoma-FISH, there was $70 \%$ overall agreement $(P=0.039)$ (Table 3b).

We also analyzed test performance for certain histologic categories in the challenging group, specifically spitzoid lesions (37 cases) and lesions with a differential diagnosis of dysplastic nevus vs nevoid melanoma, herein referred to as 'nevoid' lesions (35 cases). In relation to the histopathologic interpretation, both melanoma-FISH and myPath gene expression score showed a lower sensitivity for spitzoid lesions than for nevoid lesions (FISH: $50 \%$ vs $62 \%$; myPath $30 \%$ vs $64 \%$, respectively) (Table 4). The overall test agreement with the histopathologic interpretation was also lower for spitzoid lesion than for nevoid lesions for both tests (FISH: 67\% vs 71\%; myPath $58 \%$ vs $68 \%$, 
Table 4 Correlation of melanoma-FISH and myPath gene expression score with the expert histopathologic interpretation and interest correlation for subcategories of challenging cases

\begin{tabular}{|c|c|c|c|c|}
\hline & Sensitivity (\%) & Specificity (\%) & Agreement (\%) & No. of cases \\
\hline \multicolumn{5}{|c|}{ (A) Challenging spitzoid lesions } \\
\hline FISH vs Histology & 50 & 79 & 67 & 37 \\
\hline myPath vs Histology & 30 & 89 & 58 & \\
\hline FISH vs myPath & & & 78 & \\
\hline \multicolumn{5}{|c|}{ (B) Challenging nevoid lesions } \\
\hline FISH vs Histology & 62 & 83 & 71 & 35 \\
\hline myPath vs Histology & 64 & 75 & 68 & \\
\hline FISH vs myPath & & & 62 & \\
\hline \multicolumn{5}{|l|}{ (C) All challenging cases $^{\mathrm{a}}$} \\
\hline FISH vs Histology & 56 & 83 & 70 & $78^{*}$ \\
\hline myPath vs Histology & 52 & 80 & 64 & \\
\hline FISH vs myPath & & & 70 & \\
\hline
\end{tabular}

A—challenging spitzoid cases; B-challenging nevoid cases; $\mathrm{C}$-all challenging cases.

aThree ‘traumatized nevus vs regressed melanoma' lesions and three blue nevus-like lesions.

respectively). In contrast, the inter-test agreement was higher for spitzoid lesions compared with nevoid ones $(78 \%$ vs $62 \%)$. Of the remaining challenging lesions, three showed features in the spectrum of traumatized nevus vs regressed melanoma (1 'favor malignant', FISH-negative, myPathpositive; 2 'favor benign', both FISH-negative, 1 myPath-positive) and three were blue nevus-like (2 'favor benign' and 1 indeterminate, all FISHnegative, myPath negative).

\section{Test Performance in The Whole Cohort}

When considering the entire cohort of 117 cases, melanoma-FISH had an overall sensitivity of $69 \%$ and a specificity of $91 \%$ in relation to the histopathologic interpretation (Table 2c), while the gene expression score had a slightly lower sensitivity of $55 \%$ and a specificity of $88 \%$ (Table 2c). The two tests showed concordant results in 74 cases, discordant results in 27 cases, while 16 cases were classified as 'indeterminate' by the gene expression score, resulting in $73 \%$ overall agreement for nonindeterminate results $(P<0.001)$ (Table 3c).

\section{Combined-Test Performance}

When using a combined-test approach, where a 'positive' molecular result was considered if either FISH or myPath score was positive in any combination with the other test's result, we obtained the highest test performance when considering a molecular result as negative if either both FISH and myPath score were negative or FISH was negative with myPath score indeterminate. In this situation, the combined test showed a sensitivity of $100 \%$ and a specificity of $100 \%$ in the unequivocal group. In the challenging group the sensitivity was $70 \%$, higher than that of FISH or myPath score alone
( $56 \%, 52 \%$ ), the specificity was $70 \%$, lower than that of FISH or myPath score alone $(83 \%, 80 \%)$ and the overall agreement was $70 \%$, similar to that of FISH alone.

\section{Clinical Outcome}

We attempted to correlate the molecular test results with clinical outcome data. Given the relatively recent case pool, long-term follow-up data such as survival or metastasis development were not available for his study. Sentinel lymph node status was obtained in 23 cases, and was positive in only 7 cases, of which some showed negative molecular results by both melanoma-FISH and myPath score (data not shown). However, given the lack of longterm follow-up, and the low number of cases, a meaningful correlation of data from the two molecular tests with the clinical outcome was not possible.

\section{Evaluation of Melanoma-FISH with Low Cutoffs}

We also used a second set of less stringent melanoma-FISH cutoffs reported on commercial platforms (see Materials and Methods). With these cutoffs, 27 of the 80 initially negative cases were classified as 'borderline positive' (12 benign/favor benign, 10 ambiguous, 5 malignant/favor malignant). Over the entire cohort, the sensitivity of melanomaFISH increased when applying these lower cutoffs ( $81 \%$ from $69 \%$ ), at the expense of specificity (68\% from $91 \%$ ) (Table 5c). The same trends were observed when looking at the unequivocal group (sensitivity 93\% unchanged, specificity 79\% from $100 \%$ ) or the challenging group separately (sensitivity $74 \%$ from $56 \%$; specificity $60 \%$ from $83 \%$ ) (Table $5 a$ and b). By gene expression score, most of the FISH-'borderline positive' cases (19 of 27) were 
Table 5 Correlation of melanoma-FISH with low cutoff values (including FISH-'borderline positive' cases as positive) with histopathologic interpretation (A-unequivocal cases; B-challenging cases; C-all cases) and with myPath gene expression score (Dall cases)

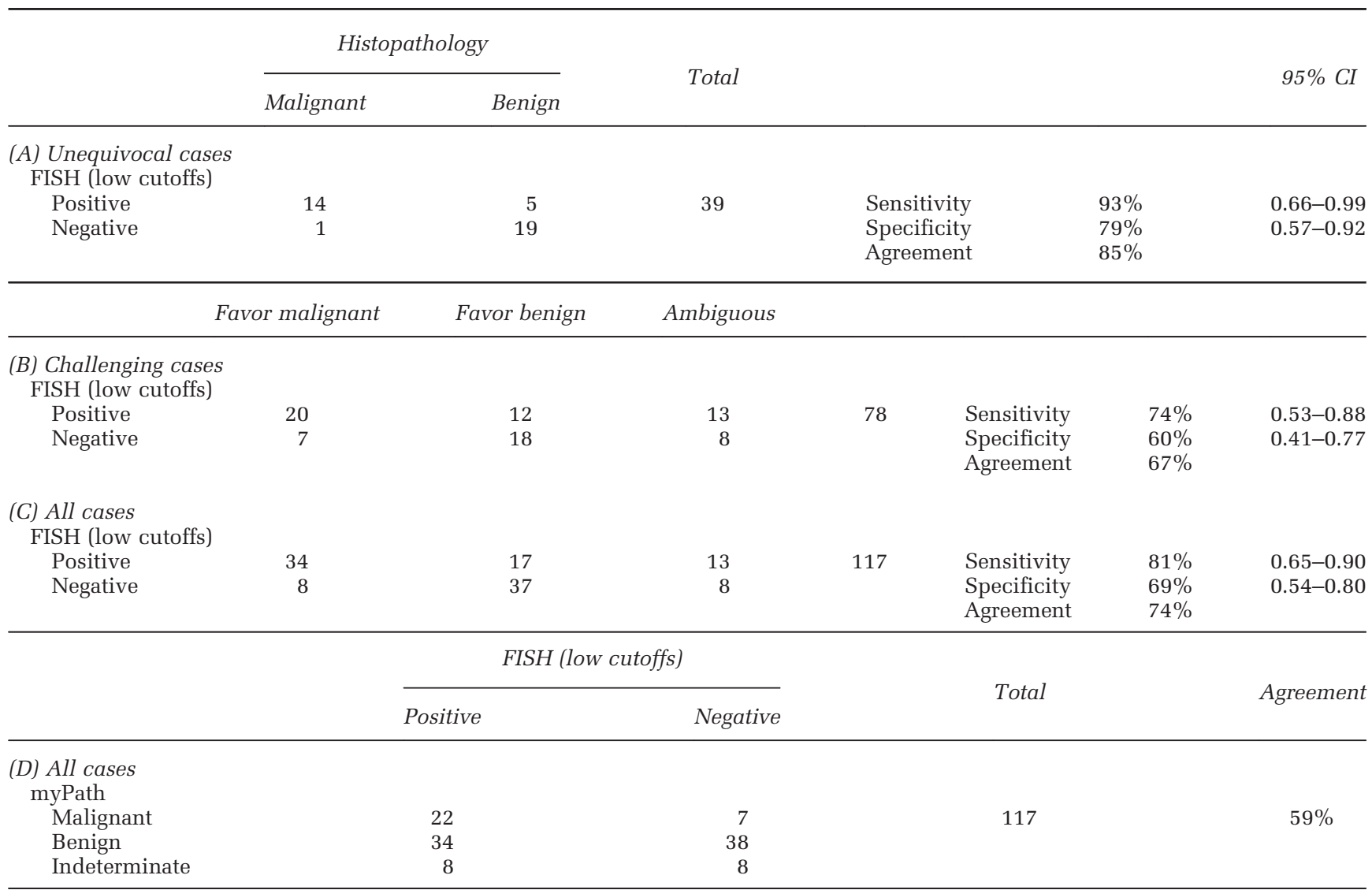

Statistic parameters calculated on non-indeterminate cases.

classified as 'benign' and the overall agreement between the two tests on the entire cohort was reduced to $59 \%$ (Table $5 \mathrm{~d}$ ).

\section{Discussion}

While accurate diagnosis of melanocytic lesions is critical for treatment and prognostic purposes, the distinction between benign and malignant lesions solely on histopathologic grounds remains a controversial and challenging task in a subset of cases. Two-thirds of the cases included in our study were interpreted as histopathologically indeterminate. (It is true though that our cohort was preselected for difficult cases.) Molecular tests such as FISH and gene expression score are now available and are deployed to provide the pathologist with additional diagnostic information in such ambiguous cases. Multi-probe melanoma-FISH targets genomic aberrations (gains or losses) at key loci frequently involved in melanoma. ${ }^{6,20}$ While the FISH assay does not correlate $100 \%$ with diagnosis for histopathologically unequivocal cases, the pathologist faced with an indeterminate melanocytic lesion is hard-pressed not to interpret it as melanoma in the presence of
FISH-specific genomic aberrations especially if already favoring melanoma. On the other hand, with a negative FISH result, a malignant process is not necessarily excluded but becomes less likely. The gene expression score assay was also developed to distinguish between benign and malignant melanocytic lesions with reported sensitivity and specificity comparable to those of FISH, based on validation studies with histopathologically unequivocal lesions, ${ }^{21,22}$ but, as with FISH, the greatest clinical utility for this test relies in its performance in morphologically ambiguous cases.

In our study, melanoma-FISH in the 5-probe format used (6p25, 11q13, 8q24, and 9p21/CEP9) showed a sensitivity of $93 \%$ and a specificity of $100 \%$ for unequivocal lesions. These parameters decreased to $56 \%$ sensitivity and $83 \%$ specificity for histopathologically challenging cases for which a benign or malignant interpretation was favored after expert review. Within the challenging cases, the sensitivity was lower for spitzoid lesions than for nevoid ones ( $50 \%$ vs $62 \%)$. Overall, these results are comparable to those of previous reports showing a significant decrease in FISH sensitivity and less so in specificity for histopathologically ambiguous 
lesions, particularly spitzoid ones, compared with unequivocal lesions. ${ }^{16,17,19,24,25}$ In an effort to preserve uniformity, FISH results obtained with 6q23/CEP6 probes, used as part of the protocol at two of the three institutions, were not reported in this study. However, it is worth mentioning that a few cases, including some 'false-negative' ones, showed FISH abnormalities only by the 6q23/CEP6 criterion, thus potentially further raising the sensitivity of a full 7-probe FISH format in the unequivocal group (data not shown).

The gene expression score showed a lower sensitivity than FISH in the unequivocal group (62\% vs $93 \%)$ and marginally lower in the challenging group ( $52 \%$ vs $56 \%$ ), while its specificity was similarly to FISH in both the unequivocal group (95\% vs $100 \%$ ) and in the challenging group (80\% vs $83 \%$ ). As with FISH, myPath sensitivity was lower for challenging spitzoid lesions than for nevoid ones (30\% vs 64\%). However, this difference in sensitivity appears more severe than for FISH. Furthermore, the performance of myPath score vs histology in nonspitzoid lesions was by-and-large on par with that of FISH. This suggests a possibly weakness of the gene expression test for spitzoid lesions. On the other hand, given that borderline spitzoid lesions uncommonly show an aggressive course, it is possible that malignancy in challenging spitzoid tumors is overdiagnosed on histologic grounds, rather than missed by the molecular tests. A critical caveat in comparing these performance metrics comes from the fact that the gene expression score includes an 'indeterminate' category (10\% of unequivocal cases, $15 \%$ of challenging cases) that cannot be accurately accounted for when calculating sensitivity, specificity, or overall agreement. ${ }^{26}$ The lack of a committing result by the gene expression score in a significant proportion of cases, seemingly higher for ambiguous lesions, is an important limitation of this assay that FISH does not have, although some commercial laboratories report a 'borderline FISH positive' result with the same caveats.

When comparing melanoma-FISH and gene expression score, we found disagreement between the two tests in approximately $27 \%$ of cases overall. Not surprisingly, the proportion of discordant results was higher in the group of lesions with challenging histopathology (30\%), compared with that of unequivocal cases $(20 \%)$. Furthermore, within the unequivocal group, the proportion of disagreement between the two tests $(20 \%)$ was greater than that between each test and the histopathologic interpretation (3\% for FISH; 17\% for gene expression score). However, for the challenging group, the inter-test disagreement $(30 \%)$ was at least the same or smaller than that between each test and histopathology $(30 \%$ for FISH; 36\% for gene expression score). Even for challenging spitzoid lesions, where both tests showed the lowest sensitivity, the inter-test disagreement $(22 \%)$ was almost as low as for unequivocal cases. A corollary of the higher inter-test agreement is that when the two tests are combined to render a single result (eg a result is positive if either FISH of myPath is positive) that is then compared with the morphologic interpretation, in the best test performance scenario, the overall agreement of a combined test is similar to that of either test alone for the challenging group. For seven challenging cases (four spitzoid, three nevoid), both molecular tests were consistent with a benign lesion, while histopathologically these lesions were interpreted as malignant. Representative image from one case with doublenegative molecular results and a positive sentinel node are shown in Figure 1. For all seven cases in question, FISH was performed at NeoGenomics Laboratories, using an automated scoring method. Applying the lower FISH cutoffs validated by this commercial lab, three of the seven cases fell under the 'borderline positive' category, including the one shown in Figure 1. While it can be argued that some of the cases with negative molecular results that were interpreted as malignant may not be considered true melanomas in the absence of outcome data, it is also possible that a small subset of 'bona fide' melanomas lacks the usual molecular alterations present in most lesions and detectable by the current molecular methods (FISH or gene expression score). These observations are in line with the findings of previous reports ${ }^{17,19,25}$ that expert histopathologic evaluation has a higher sensitivity and specificity than any currently available ancillary test for the diagnosis of melanocytic lesions.

When analyzing the 27 cases with discordant molecular test results, most of the cases (15/27, $56 \%$ ) had a positive FISH but a negative gene expression score. Two examples are shown in Figure 2. Among these, histopathologic assessment determined 10 cases to be best classified as malignant/favor malignant, 3 cases as benign/favor benign and 2 cases as ambiguous. Five of the 15 cases had spitzoid histopathologic features and tetraploidy was suspected in two cases to account for the positive FISH results. In addition, it is possible that biologic particularities in some melanomas, such as suppressed immune recognition of the tumor with under-expression of targeted immunity-related gene signatures, cause false-negative gene expression scores. The remaining 12 of the 27 cases with discordant molecular results had a negative FISH and a positive gene expression score. Two examples are shown in Figure 3. Histopathologic assessment determined that five cases were best classified as malignant, five cases as favor benign and two cases as ambiguous. Of the benign/favor benign lesions, two showed a prominent inflammatory component, which may have contributed to a false-positive gene expression score (Figure 3a). In our experience, we have encountered histopathologically unequivocal benign nevi associated with acute inflammation in the form of abscess or folliculitis, which were classified as 'malignant' by gene expression score (data not shown). This is likely a reflection of the 
reliance of the myPath assay on the expression of several genes with known roles in immune regulation. Alternatively, in lesions with a prominent inflammatory infiltrate, one could also argue that a false-negative FISH result may occur by artifactual counting of FISH-normal nuclei from inflammatory
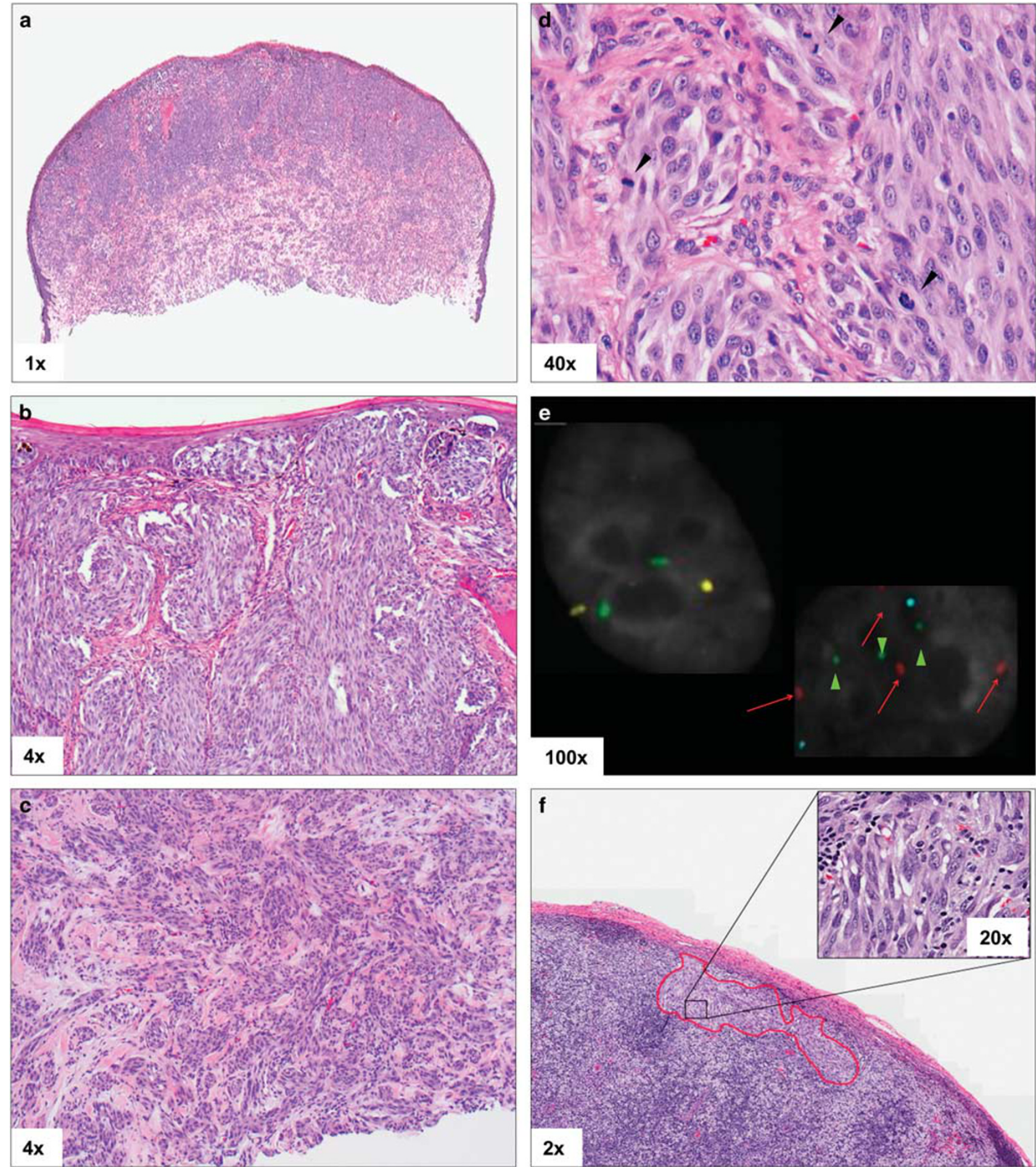

Figure 1 Representative images of a case with sentinel lymph node metastases with negative ('borderline') melanoma-FISH and negative gene expression score. A pigmented lesion on the left abdomen of a 9-year-old boy showed an atypical intradermal melanocytic proliferation (a) with focal epidermal involvement (b). The melanocytes exhibited a spindled morphology without maturation with dermal descent, significant nuclear atypia (c) and multiple deep mitotic figures (d, arrow heads). The histopathologic interpretation was 'malignant'. Melanoma-FISH (NeoGenomics) showed 'borderline' gains of 6p25 'red arrows' and 11q13 'green arrow heads' (e). The sentinel lymph node biopsy showed metastatic melanoma in the highlighted area (f) (inset: high power of the metastasis area). 
cells (Figure 3). In cases of thin melanoma, FISH is difficult to score, potentially generating falsenegative results due to lesion size and distribution, while these factors would not affect the gene expression score assay and appeared to have contributed to discrepant results in one case in our
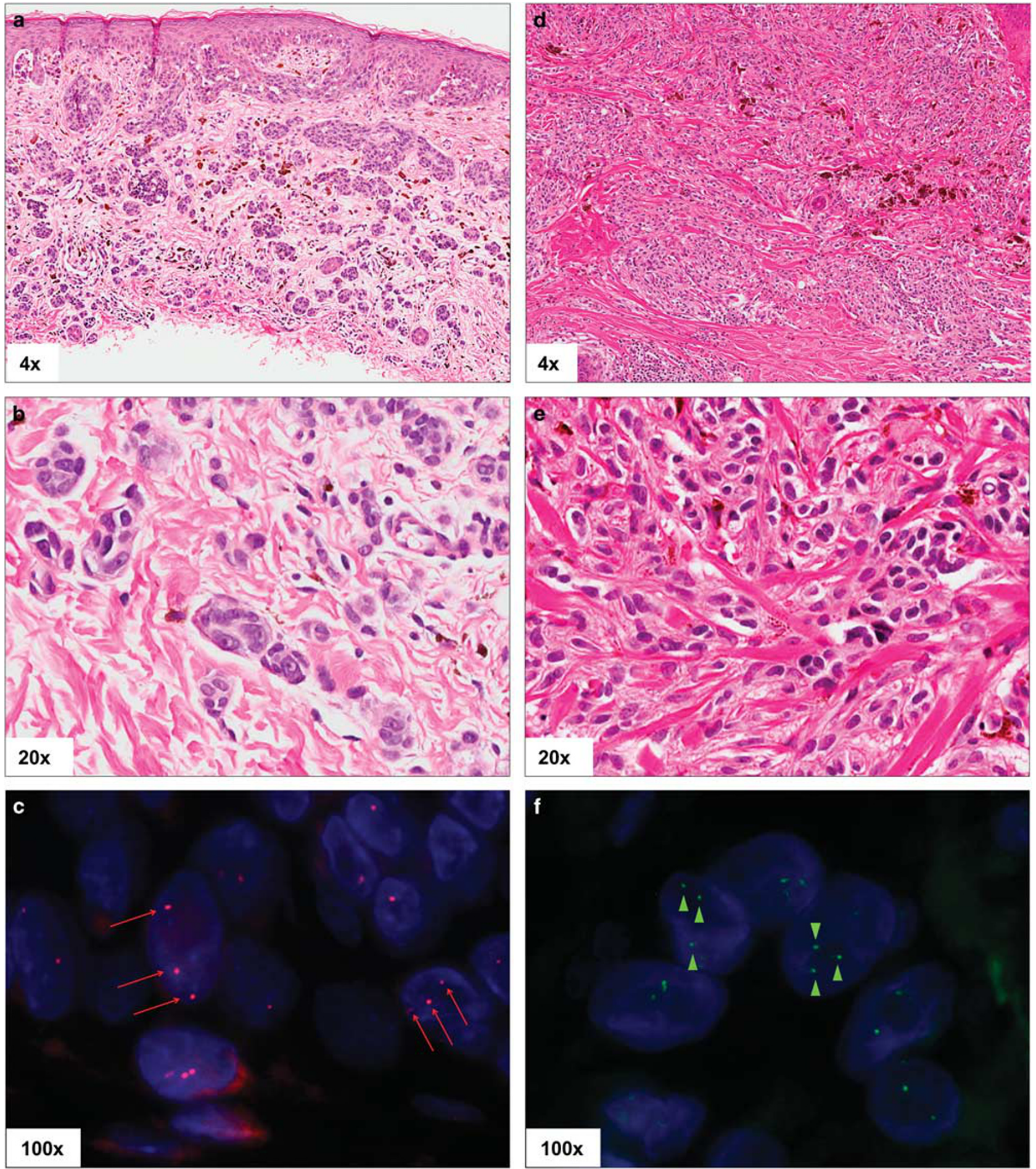

Figure 2 Representative images of two cases with positive melanoma-FISH and benign gene expression score (a, b, d, e: hematoxylineosin; c, f: FISH). One case was a pigmented flat lesion from the left abdomen of an 18-year-old woman, showing histologic asymmetry with bridging of junctional nests, dermal melanophages and nests of atypical epithelioid melanocytes without maturation (a, b). FISH showed gain of 6 p25 (c, red signals). The histopathologic interpretation was 'favor malignant'. The second case was a pigmented elevated lesion from the parietal scalp of a 57-year-old woman, showing epithelioid to spindled cells in nests, mild cytologic atypia, and tracking on the hair follicle (d, e). FISH showed gain of 11q13 (f, green signals). The histopathologic interpretation was 'favor malignant'. 

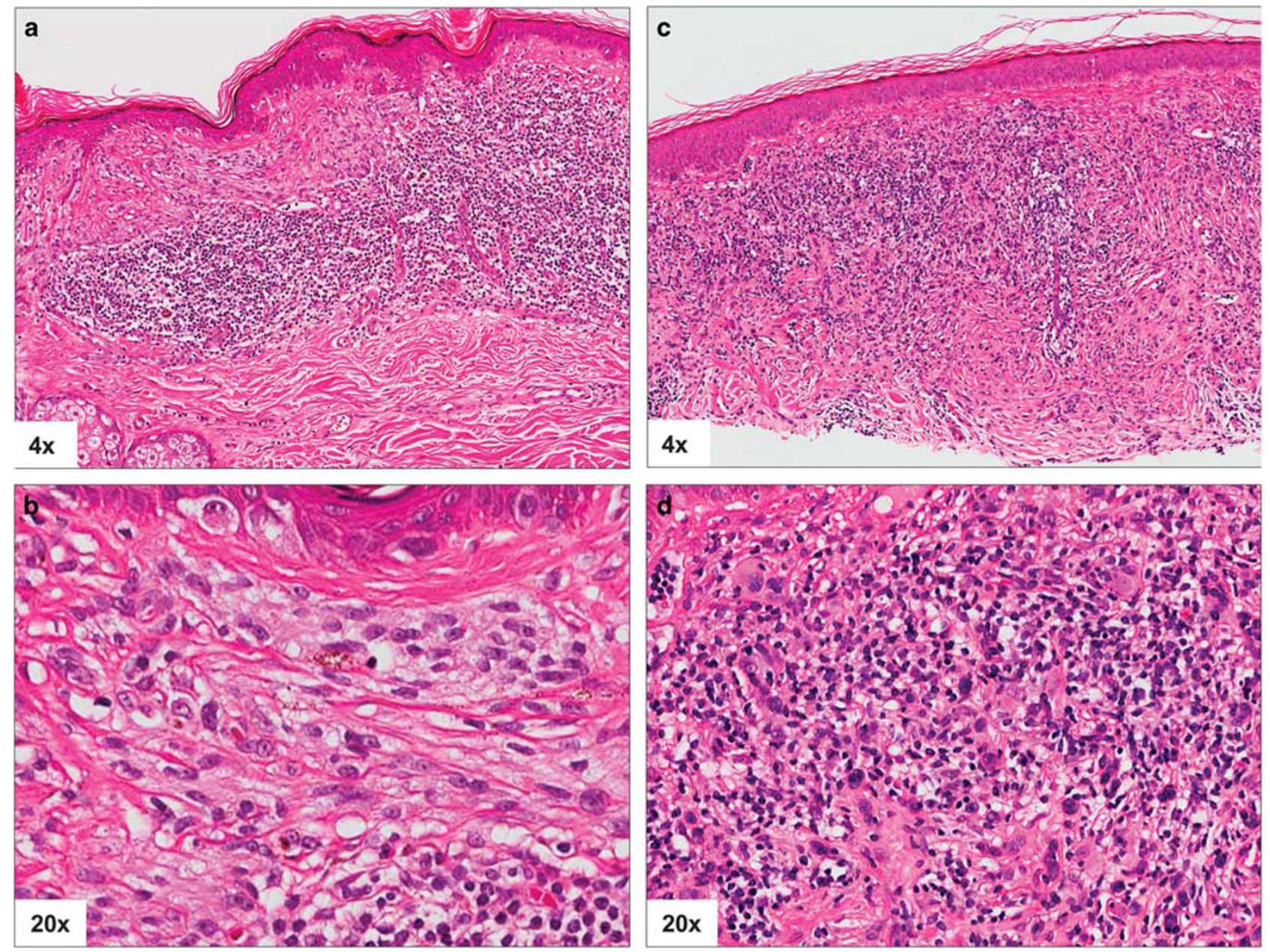

Figure 3 Representative images of two cases with negative melanoma-FISH and malignant gene expression score (a-d: hematoxylineosin). One case was pigmented flat lesion on the right shoulder of a 66-year-old man, showing an atypical compound melanocytic proliferation with junctional nest bridging, fibroplasia, some cytologic atypia, and prominent inflammation (a, b). The histopathologic interpretation was 'benign'. The second case was a pigmented raised lesion from the mid back of a 54-year-old woman showing an inflamed atypical proliferation of single epithelioid melanocytes with large vesicular nuclei, prominent nucleoli and occasional multinucleation associated with prominent inflammation (c, d). The histopathologic interpretation was 'malignant'.

cohort. The presence of an associated or precursor nevus may also contribute to discrepant results between the two tests, either by scoring benign melanocyte nuclei in the FISH assay as described above, or by a diluting effect in the gene expression score assay. Indeed, in our experience, we have encountered cases of melanomas with a predominant precursor nevus for which the gene expression score was negative or indeterminate. In theory, the FISH assay has the advantage of allowing assessment of cell morphology and distinction of atypical melanocytes for the experienced, whereas the myPath score analyzes a pool of cells and in our study whole tissue sections were used for this assay. However, the sensitivity of the gene expression score assay may be improved by incorporating a microdissection step. Another cause for potential discrepancies between FISH and the myPath score is the difference in the material used for the two tests: ${ }^{27}$ in particular, deeper levels used for one test may not be entirely representative of the lesion in the sections on which the second test is performed. We compared $\mathrm{H} \& \mathrm{E}$ sections on several of our cases cut before and after the levels used for each of the two tests and did not observe a significant difference in the lesion volume or histopathologic features.

Studies of melanoma-FISH on challenging cases using the cutoffs originally established on unequivocal lesions have suggested that the test has a relatively low sensitivity and a high specificity compared with expert histopathologic interpretation and clinical outcome. ${ }^{16,17}$ In an attempt to increase the test's sensitivity, NeoGenomics Laboratories, one of the commercially available platforms, created set of less stringent melanoma-FISH cutoffs with their commercial FISH assay (see Materials and Methods) (http://www.neogenomics.com/neosite-melanoma.htm). This creates a 'borderline positive' category of cases, which, as stipulated by the developer, should be interpreted 'within the context of other pathology 
and clinical data'. In our study, while the low cutoffs captured five cases interpreted as melanoma/favor melanoma, they also flagged 11 benign/favor benign cases, resulting in a decrease in specificity (91-69\% overall) greater than the increase in sensitivity (69-81\% overall). Furthermore, the gene expression score classified the majority of FISH-'borderline positive' cases $(19 / 27,70 \%)$ as 'benign'. These results suggest that the utility of the 'borderline positive' FISH category is not in helping the pathologist to reach a diagnosis of melanoma but to guide a more aggressive therapeutic approach for lesions with a benign/favor benign histopathologic appearance.

Clinical behavior data such as sentinel lymph node status, development of metastases, or survival have been used in some previous studies as gold standard parameters to assess the accuracy of melanoma-FISH for ambiguous lesions, ${ }^{16,17,19}$ although this likely represents too narrow a definition of melanoma. As a limitation to our study, the case cohort was comprised of recent biopsies, thus not allowing any analysis based on survival or disease progression. While the sentinel lymph node status was available in a subset of cases and even positive in a minority, the value of this information as surrogate for biologic behavior is uncertain in the absence of long-term follow-up, especially for challenging and ambiguous lesions. While statistical correlations were not possible, the fact that test results discordant with the sentinel node status were obtained with both molecular tests in some cases, underscores the challenge in accurately classifying these cases, for which long-term outcome may be the only reliable indicator of disease biology.

In conclusion, melanoma-FISH gene and myPath expression score are valuable ancillary tools, though both with limitations and disagreement with each other and with the expert histopathologic interpretation in a subset of cases. The results provided by these tests can be useful in challenging cases when used in the conjunction with the clinical and histopathologic findings. Further studies with clinical outcome data are warranted to compare the accuracy of these methods for the classification of melanocytic lesions.

\section{Disclosure/conflict of interest}

SDB serves as a consultant on the Advisory Board of Myriad Genetic Laboratories. The remaining authors declare no conflict of interest.

\section{Disclaimer}

This research was performed entirely with internal resources without support from external sources or industry.

\section{References}

1 Siegel RL, Miller KD, Jemal A. Cancer statistics, 2015. CA Cancer J Clin 2015;65:5-29.

2 McGinnis KS, Lessin SR, Elder DE et al. Pathology review of cases presenting to a multidisciplinary pigmented lesion clinic. Arch Dermatol 2002;138: 617-621.

3 Corona $\mathrm{R}$, Mele A, Amini $\mathrm{M}$ et al. Interobserver variability on the histopathologic diagnosis of cutaneous melanoma and other pigmented skin lesions. J Clin Oncol 1996;14:1218-1223.

4 Scolyer RA, Shaw HM, Thompson JF et al. Interobserver reproducibility of histopathologic prognostic variables in primary cutaneous melanomas. Am J Surg Pathol 2003;27:1571-1576.

5 Lodha S, Saggar S, Celebi JT et al. Discordance in the histopathologic diagnosis of difficult melanocytic neoplasms in the clinical setting. J Cutan Pathol 2008;35: 349-352.

6 Gerami P, Jewell SS, Morrison LE et al. Fluorescence in situ hybridization (FISH) as an ancillary diagnostic tool in the diagnosis of melanoma. Am J Surg Pathol 2009;33:1146-1156.

7 Gerami P, Busam KJ. Cytogenetic and mutational analyses of melanocytic tumors. Dermatol Clin 2012;30: $555-566 \mathrm{v}$.

8 Gammon B, Gerami P. Fluorescence in situ hybridization for ambiguous melanocytic tumors. Histol Histopathol 2012;27:1539-1542.

9 Busam KJ, Fang Y, Jhanwar SC et al. Distinction of conjunctival melanocytic nevi from melanomas by fluorescence in situ hybridization. J Cutan Pathol 2010;37:196-203.

10 Dalton SR, Gerami P, Kolaitis NA et al. Use of fluorescence in situ hybridization (FISH) to distinguish intranodal nevus from metastatic melanoma. Am J Surg Pathol 2010;34:231-237.

11 Gammon B, Beilfuss B, Guitart J et al. Fluorescence in situ hybridization for distinguishing cellular blue nevi from blue nevus-like melanoma. J Cutan Pathol 2011;38:335-341.

12 Gerami P, Mafee M, Lurtsbarapa T et al. Sensitivity of fluorescence in situ hybridization for melanoma diagnosis using RREB1, MYB, Cep6, and 11q13 probes in melanoma subtypes. Arch Dermatol 2010;146: 273-278.

13 Newman MD, Lertsburapa T, Mirzabeigi $\mathrm{M}$ et al. Fluorescence in situ hybridization as a tool for microstaging in malignant melanoma. Mod Pathol 2009;22:989-995.

14 Pouryazdanparast P, Haghighat Z, Beilfuss BA et al. Melanocytic nevi with an atypical epithelioid cell component: clinical, histopathologic, and fluorescence in situ hybridization findings. Am J Surg Pathol 2011;35:1405-1412.

15 Pouryazdanparast $\mathrm{P}$, Newman $\mathrm{M}$, Mafee $\mathrm{M}$ et al. Distinguishing epithelioid blue nevus from blue nevus-like cutaneous melanoma metastasis using fluorescence in situ hybridization. Am J Surg Pathol 2009;33:1396-1400.

16 Gaiser T, Kutzner H, Palmedo G et al. Classifying ambiguous melanocytic lesions with FISH and correlation with clinical long-term follow up. Mod Pathol 2010;23:413-419.

17 Vergier B, Prochazkova-Carlotti M, de la Fouchardiere A et al. Fluorescence in situ hybridization, a diagnostic 
aid in ambiguous melanocytic tumors: European study of 113 cases. Mod Pathol 2011;24:613-623.

18 Massi D, Cesinaro AM, Tomasini C et al. Atypical Spitzoid melanocytic tumors: a morphological, mutational, and FISH analysis. J Am Acad Dermatol 2011;64:919-935.

19 Tetzlaff MT, Wang WL, Milless TL et al. Ambiguous melanocytic tumors in a tertiary referral center: the contribution of fluorescence in situ hybridization (FISH) to conventional histopathologic and immunophenotypic analyses. Am J Surg Pathol 2013;37: 1783-1796.

20 Gerami P, Li G, Pouryazdanparast P et al. A highly specific and discriminatory FISH assay for distinguishing between benign and malignant melanocytic neoplasms. Am J Surg Pathol 2012;36: 808-817.

21 Clarke LE, Bess E, Evans B et al. A clinically validated gene expression score impacts diagnosis and management recommendations of melanocytic lesions by dermatopathologists. ASDP Annual Meeting abstract, 2014.

Supplementary Information accompanies the paper on Modern Pathology website (http://www.nature.com/ modpathol)
22 Clarke LE, Warf BM, Flake DD II et al. Clinical validation of a gene expression signature that differentiates benign nevi from malignant melanoma. J Cutan Pathol 2015;42:244-252.

23 Moore MW, Gasparini R. FISH as an effective diagnostic tool for the management of challenging melanocytic lesions. Diagn Pathol 2011;6:76.

24 North JP, Garrido MC, Kolaitis NA et al. Fluorescence in situ hybridization as an ancillary tool in the diagnosis of ambiguous melanocytic neoplasms: a review of 804 cases. Am J Surg Pathol 2014;38:824-831.

25 Zembowicz A, Yang SE, Kafanas A et al. Correlation between histologic assessment and fluorescence in situ hybridization using MelanoSITE in evaluation of histologically ambiguous melanocytic lesions. Arch Pathol Lab Med 2012;136:1571-1579.

26 Simel DL, Feussner JR, DeLong ER et al. Intermediate, indeterminate, and uninterpretable diagnostic test results. Med Decis Making 1987;7:107-114.

27 Garib G, Ariano E, Quirey $\mathrm{R}$ et al. myPathTM (melanoma assay) in our practice: is it helpful? ASDP Annual Meeting abstract, 2014. 